Relmecs, diciembre 2017, vol. 7, no. 2, e027, ISSN 1853-7863

Universidad Nacional de La Plata. Facultad de Humanidades y Ciencias de la Educación. Centro Interdisciplinario de Metodología de las Ciencias Sociales.

Red Latinoamericana de Metodología de las Ciencias Sociales

\title{
La refutación del romanticismo y las condiciones epistémicas para la investigación socio-histórica en el neoilluminismo de Nicola Abbagnano
}

\author{
The Confutation of Romanticism and the epistemic conditions for a \\ socio-historic investigation of Nicola Abbagnano's Neo-Enlightment
}

\author{
Diego Alfredo Pérez Rivas * \\ * CONACYT, México | diego.perez.rivas@ucm.es
}

\section{PALABRAS CLAVE \\ Historia \\ Sociología \\ Idealismo}

Positivismo

Neoilluminismo

\section{RESUMEN}

Una de las disputas filosóficas más interesantes acerca del status epistémico de la historia y la sociología estuvo protagonizada por el idealismo y el positivismo. En la primera parte se propone una breve reconstrucción de este debate. En la segunda parte, se analiza la crítica de Abbagnano a ambos modelos, al ser considerados sistemas románticos. En la tercera y cuarta parte, se analizan las condiciones epistémicas propuestas por el filósofo italiano para la historia y la sociología, resaltando la importancia de su refutación del romanticismo. Finalmente, se evalúa si el esquema de Abbagnano es completo.

KEYWORDS

History

Sociology

Idealism

Positivism

\section{ABSTRACT}

One of the most interesting philosophical debate about the epistemic status of history and sociology emerged from the opposition between idealism and positivism. The first part of this article offers a brief description of this debate. The second part analyses Abbagnano's critique of the two models, both regarded as romantic models. The third and fourth sections focus on the epistemic conditions for history and sociology advanced by the Italian philosopher, and highlight the importance of the confutation of Romanticism. Finally, the last section assesses if Abbagnano's framework is complete.

Neo-Enlightment 
Los estudios más recientes sobre historia de la filosofía italiana, reconocen casi unánimemente la existencia de un período hegemónico del modelo idealista durante la segunda mitad del s. XIX y la primera del s. XX. La supremacía de tal paradigma produjo un fenómeno de hermetismo y aislamiento que condicionó el diálogo de la cultura filosófica italiana con las culturas filosóficas extranjeras. Como consecuencia de este hecho, la introducción de la sociología en el sistema educativo italiano fue tardía respecto a Francia, Inglaterra, Alemania y Estados Unidos. En este sentido, el movimiento neoilluminista y, en particular, el pensamiento filosófico de Abbagnano tuvieron un papel relevante. En el presente artículo se propone una revisión crítica de la filosofía de Abbagnano resaltando su importancia para: (1) la introducción de la sociología empírica en la cultura filosófica italiana y (2) la elaboración de un conjunto de condiciones epistémicas para la sociología y la historia. En el artículo se explica la relevancia que tuvo la refutación del romanticismo y del determinismo en la construcción de un modelo condicionalista para las ciencias sociales.

\section{Idealismo vs positivismo: el status epistémico de la sociología y la historia}

Desde la segunda mitad del s. XVIII, la disputa filosófica entre idealismo y positivismo condicionó la configuración del panorama intelectual europeo. En el caso específico de la cultura filosófica italiana, caracterizada por un largo período de aislamiento, el debate se desarrolló en la primera mitad del Novecento. Hasta ese momento las tesis del positivismo francés habían producido un enorme impacto en el ambiente cultural continental. Esta corriente sostenía que las especulaciones metafísicas promovidas por el idealismo debían ser sustituidas por experimentos empíricos o por demostraciones matemáticas rigurosas para sentar las bases de las distintas disciplinas científicas. El triunfo de la industria como consecuencia del desarrollo de las ciencias empíricas abanderó este empeño positivista. El neoidealismo, en cambio, desarrolló una intensa crítica metafísica que implicaba el estatus epistémico del conocimiento obtenido mediante procedimientos empíricos.

La disputa entre idealismo y positivismo puede considerarse un punto clave para el desarrollo de la sociología y la historiografía modernas, pero abarcó también otras disciplinas del conocimiento. En este cauce, la filosofía positivista se caracterizó por la búsqueda de un programa enciclopédico capaz de unificar a las ciencias básicas mediante las matemáticas, así como por su propuesta de construir nuevos horizontes de investigación empírica para las ciencias sociales, poniendo en entredicho las tesis del idealismo. Considerada por Comte como el producto de un proceso de superación de las indagaciones metafísicas y especulativas, la filosofía positiva se inspiró en el iluminismo, revalorando el papel de las metodologías experimentales y el análisis matemático en la construcción del conocimiento epistémicamente riguroso. En su Curso de filosofía positiva Comte explicó:

En el estado actual del desarrollo de nuestros conocimientos positivos es interesante ver la matemática menos como una parte constituyente de la filosofía propiamente dicha que como la verdadera base fundamental de toda esta filosofía, por lo menos después de Descartes y Newton, aunque hablando debidamente sea lo uno y lo otro (2004, p. 117).

El idealismo y el positivismo esbozaron proyectos mutuamente excluyentes para la elaboración del conocimiento epistémicamente riguroso. Mientras el idealismo consideraba el saber epistémico como un tipo de conocimiento especulativo, apodíctico y referido a esencias o sustancias, el positivismo lo consideraba el resultado de procesos inductivos y comparativos (las metodologías experimentales o formales) en los que se obtienen conocimientos parciales, pero interconectados e interdependientes, de la realidad. En este sentido, es sobresaliente la vinculación propuesta por Comte entre (a) las ciencias naturales abstractas, que tienen como objeto el descubrimiento de las leyes que gobiernan los fenómenos, y (b) las ciencias naturales particulares, que buscan la aplicación de tales leyes a la historia efectiva de los diferentes seres existentes (2004, p. 82).

La oposición del positivismo contra el idealismo no involucró exclusivamente la noción de saber epistémico o el problema de la unificación de las ciencias. Mientras el positivismo buscaba dotar a la sociología tanto de un lenguaje auténtico como de una metodología particular para que pudiera liberarse de la filosofía, el idealismo rechazaba absolutamente este divorcio al sostener que la lógica y la ética pura (eticidad) coincidían en una teoría normativa del comportamiento social. 
En el fondo, ambos modelos edificaron arquitecturas conceptuales diferentes tanto para la interpretación de los fenómenos sociales como para la puesta en práctica de dichos conocimientos. El positivismo, siendo heredero de una tradición particularmente empirista, buscaba responder a la pregunta del cómo de los fenómenos sociales, recurriendo a una metodología prioritariamente descriptiva, pero en la que la filosofía y las matemáticas tendrían una función unificadora de los saberes particulares. Esta pretensión positivista implicaba, en cierto sentido, la igualación entre momento descriptivo y momento prescriptivo. Como explica Massimo Mori:

la naturaleza nomológica de estas filosofías (positivistas) absorbe tanto la exigencia científico-descriptiva como la ético prescriptiva, ya que la ley se identifica con la norma: lo que sucede necesariamente es también lo que debe suceder, de modo que se consigan determinados fines (2010, p. 89).

El idealismo, en cambio, siendo heredero de una tradición racionalista buscaba explicar el porqué de los fenómenos sociales, recurriendo a una metodología de carácter prescriptivo o normativo que pretendía condicionar la vida social.

Otra distinción fundamental consistió en que mientras el positivismo favoreció el uso del lenguaje matemático como eje del conocimiento científico, el idealismo se inspiraba en un lenguaje metafísico-especulativo. La apuesta del positivismo consistió en establecer los principios que posibilitarían la autonomía de la sociología, inspirándose en el modelo de la física, esto es, poniendo énfasis en el estudio de magnitudes. La pretensión del modelo positivista consistió en reemplazar los modelos filosófico-especulativos y jurídicos-normativos que había implantado el idealismo para la interpretación de los fenómenos sociales, apostando por la mensurabilidad de los fenómenos. Por lo mismo, el positivismo trató de consolidar el divorcio de la sociología empírica tanto de la doctrina jurídica como de la filosofía política, algo que el idealismo no estaba dispuesto a conceder.

La disputa entre positivismo e idealismo involucró también una clara diferencia respecto a la aplicación del conocimiento para la organización social. El positivismo pretendía impulsar, mediante la organización y unificación del conocimiento, la modernización industrial. Mientras que el idealismo, profundamente anticientífico y tradicionalista, consideraba a las metodologías empíricas como productoras de pseudoconocimientos. El positivismo defendió, por tanto, un modelo de sociedad inspirado en la ciencia y en la técnica, mientras que el idealismo defendió un modelo que priorizaba el papel del arte, la religión y la filosofía especulativa. Veámoslo en detalle.

En la filosofía hegeliana la única forma de acceder a la verdad es mediante un método dialéctico-especulativo en donde la doctrina del concepto es el aspecto más relevante (Hegel, 1974, pp. 160-164). En el caso específico del idealismo italiano, tanto Croce como Gentile partieron de premisas similares. Para el primero, solamente la lógica permite el conocimiento conceptual-universal, mientras que las metodologías empíricas son consideradas actividades que producen pseudoconceptos (1902, p. 33). Para el segundo, "la ciencia, en cuanto particular y asistemática está, respecto a la realidad, en la posición del arte, que no es filosofía porque su realidad es una realidad particular y, por tanto, puramente subjetiva” (1944, p. 200).

En oposición al empirismo, el idealismo elaboró un proyecto especulativo en el que la prioridad estaba puesta en la teoría, no en la observación ni en la experimentación. En este sentido, la filosofía hegeliana abordó el problema de la posibilidad de estudiar los fenómenos sociales a partir de una metodología racionalista y normativa, en lo que se conoce como filosofía del espíritu.

La filosofía del espíritu, en el pensamiento hegeliano, se divide en tres grandes partes. En primer lugar, (1) el espíritu subjetivo. En su Enciclopedia de las Ciencias Filosóficas: (1.1) la antropología, (1.2) los estudios sobre la conciencia y (1.3) la psicología teórica, constituían sus áreas de investigación, rechazando las metodologías empíricas. En tal esquema (1.1) estudiaría el alma mediante el método dialéctico con la misión de explicar la sensación y los hábitos. A (1.2) le correspondía desentrañar los secretos de la conciencia sensible, la percepción y el intelecto, además de los fenómenos relacionados con la autoconciencia y la razón. Finalmente, (1.3) debía considerar las facultades y los modos universales de actividad del espíritu, entre los que destacan fenómenos como 
la intuición, la representación, la imaginación, la memoria, el pensamiento, el espíritu práctico y la libertad (Hegel, 1974, p. 306). En palabras de Hegel, su proyecto filosófico intentaba despertar la inteligencia de los escritos aristotélicos acerca Del alma, en oposición a la psicología empírica que priorizaba la observación y la experimentación (Hegel, 1974, p. 267).

En (2) el espíritu objetivo, Hegel consideraba la realización del espíritu libre mediante instituciones históricas concretas. Primeramente (2.1) el derecho abstracto, en el que el sujeto es la persona, entendida como el propietario abstractamente considerado. Después (2.2) la moral, en la que el sujeto posee una voluntad particular manifestándose (2.2.1) internamente como intención y (2.2.2) externamente como acción. Finalmente, (2.3) la eticidad es entendida como la superación de todas las distinciones: (2.3.1) como familia en el momento natural de la unión sexual, (2.3.2) en la sociedad civil, en tanto que sistema de satisfacción de necesidades mediante el mercado (economía clásica), la administración de justicia y la creación de la policía, y (2.3.3) como Estado, en el que se realiza la unificación de la sociedad civil y la familia, a través de tres mecanismos. Primero (2.3.3.1) la Constitución o el derecho interno; después, (2.3.3.2) el Derecho externo, y finalmente (2.3.3.3) la historia del mundo.

En el idealismo hegeliano, los fenómenos sociales son considerados como el resultado de un proceso histórico racional en el que los datos empíricos son irrelevantes, mientras que lo importante son las fuerzas o procesos a través de los cuales se manifiesta, hipotéticamente, la racionalidad intrínseca de los acontecimientos históricos. En este sentido, en (3) el momento del espíritu absoluto: (3.1) el arte, (3.2) la religión revelada y (3.3) la filosofía fueron consideradas actividades en las que el conocimiento se manifestaría de modo absoluto. En (3.1) como intuición sensible, en (3.2) como representación y en (3.3) como forma pura del concepto.

Para Hegel, las ciencias sociales se enmarcaban en el momento del espíritu objetivo (2), especialmente en la eticidad, si bien relacionándose de modo indirecto con el espíritu subjetivo y con el espíritu absoluto. Se podría decir que (1) representaba el momento de las condiciones antropológicas, psicológicas y cognitivas de posibilidad de los fenómenos sociales, mientas que (3) representaba el momento de los efectos o manifestaciones de lo absoluto en la historia. En tal cauce, Hegel concibió la filosofía del derecho como la piedra angular en la que el derecho abstracto (2.1) era la afirmación y limitación externa de la voluntad mediante la institución de la propiedad privada. Como su contraparte, la moralidad (2.2) sería la afirmación y limitación interna de la voluntad, en la que la inteligencia se impone una serie de fines y acciones. Finalmente, en la eticidad (2.3), las relaciones sociales surgirían con plena potencia en tanto que (2.3.1) sociabilidad natural o familiar, mediante el matrimonio, el patrimonio y la educación paterna: en (2.3.2) la sociedad civil, mediante la satisfacción de las necesidades descritas por las leyes del mercado enunciadas por la economía clásica, la administración de la justicia, la obra de la policía y de las corporaciones.

La filosofía idealista de Hegel, en este punto, puede considerarse como parcialmente compatible con los postulados de la economía clásica. Es importante notar que la compatibilidad de la teoría económica de Smith con el idealismo hegeliano parece estar anclada en la aceptación del escenario ideal en el que funcionaría, hipotéticamente, la economía de mercado. Sin embargo, es incompatible en lo que respecta al modelo del escocés en lo que se refiere a la inducción de datos empíricos o su teoría de los sentimientos morales. Resulta necesario recordar que el modelo económico de Smith proponía una concepción de la sociedad civil compatible con la elaborada por Hegel, es decir, entendida como un sistema de satisfacción de necesidades, la cual sería gobernada por una especie de racionalidad absoluta representada en la idea de la mano invisible. Dicha mano invisible existiría en el mundo real como una especie de gran atractor que porta constantemente a los sistemas económicos a un estado de equilibrio ideal. A eso se puede agregar que la teoría categorial de las clases sociales en Hegel no es compatible con la concepción empírica de Smith, pues en el idealismo tal tipología solamente comprendía: (a) la clase social sustancial o agrícola; (b) la clase industrial dedicada al artesanado, las fábricas y el comercio; y (c) la clase universal, protegida con el patrimonio estatal y dedicada a las labores referentes al espíritu absoluto.

Otro elemento que caracterizó al modelo idealista fue la defensa de la división radical entre ciencias de la 
naturaleza y ciencias del espíritu. Dicha división se fundaba en dos intuiciones arbitrarias e injustificadas. En primer lugar, en la idea de que las leyes que gobiernan el mundo natural (mecanismo y quimismo) no implican los fenómenos humanos y sociales (teleológicos). En segundo lugar, en la idea de que las ciencias empíricas son incapaces de adquirir conocimientos epistémicamente sólidos para explicar los fenómenos relacionados con la conciencia y la autoconciencia. De tal modo, los hechos empíricos analizados por la mecánica y la física (inorgánica y orgánica) son considerados primitivos e inferiores respecto a los conocimientos adquiridos a través del arte, la religión y la filosofía, en tanto que ciencias del espíritu absoluto. En el fondo, la postura idealista negaba la fiabilidad de los conocimientos adquiridos empíricamente, a los que concebía como intrínsecamente reduccionistas. Los idealistas consideraban que solamente los conocimientos obtenidos especulativamente podrían identificar espíritu con realidad ética (Hegel, 2003, p. 259). La discontinuidad que el idealismo trazó entre ciencias naturales y ciencias del espíritu impedía, de tal forma, un análisis científico serio sobre las bases biológicas del comportamiento humano, ya sea en su carácter individual o social.

En el idealismo, la sociología como estudio empírico de la sociedad no tenía sentido, ya que las ciencias del espíritu y la economía clásica desentrañarían mediante sus instrumentos todos los secretos de la sociabilidad racional. A diferencia de las ciencias empíricas que buscan ser descriptivamente rigurosas, imparciales y no valorativas, las ciencias del espíritu promovidas por el idealismo son prescriptivas, normativas y valorativas. Esto resulta claro si consideramos que el modelo de la economía clásica es ideográfico, dado que el escenario ideal en el que se inspira describe el funcionamiento típico de la sociedad capitalista y burguesa. Asimismo, el concepto idealista de la historia como proceso intrínsecamente racional que es gobernado por leyes universales y progresivas es el último escalafón de esta metodología especulativa, pues, en palabras de Hegel:

La providencia divina es la sabiduría que con potencia infinita realiza sus fines, esto es, el fin absoluto, racional, del mundo: y la razón es el pensamiento que se determina a sí mismo en plena libertad, el Nous (2001, p. 19).

Al igual que el idealismo, el positivismo veía en la historia la existencia de un proceso racional de carácter evolutivo. Considerada como un proceso orgánico que adquiere complejidad gradualmente, la historia daría evidencia del desarrollo de la razón humana en distintas fases de perfeccionamiento. El positivismo de Comte consideraba que existía una ley de estadios históricos que describía la historia de la humanidad como un proceso evolutivo que implica el nivel de comprensión de la realidad. Hipotéticamente, en el estado teológico, el ser humano permanecía en un estado de completa indefensión al explicar los fenómenos naturales mediante fantasiosas creencias fetichistas, politeístas o monoteístas. En el estado metafísico se construirían ontologías especulativas ampliamente infructuosas por su carencia de metodologías rigurosas y por su incapacidad predictiva. El estado positivo se alcanzaría solamente cuando la inteligencia humana lograra emanciparse de la superstición, mediante la ciencia y la técnica. En palabras de Comte, lo positivo designaría lo real en oposición a lo quimérico; lo útil en oposición a lo inútil; la certeza en oposición a lo incierto; lo preciso en oposición a lo vago; lo organizado en oposición a lo no organizado.

En el esquema de las ciencias del positivismo, las metodologías empíricas y las demostraciones matemáticas adquieren un valor relevante como modos de verificación de las teorías. La astronomía, la física, la química y la biología fueron consideradas como ciencias positivas por la rigurosidad de sus métodos y por la objetividad de sus previsiones. En la base de la búsqueda de la objetividad estaba el lenguaje matemático, considerado el hilo conductor y la base común sobre la que descansaban las diferentes ciencias positivas.

La sociología encontró en el esquema positivista un primer impulso para buscar su afirmación e independencia, mediante la búsqueda de su propio método. Gracias al proyecto de Comte de crear una física social dividida en estática social y dinámica social, la sociología podría encontrar un lenguaje para interpretar científicamente los fenómenos de la vida pública. De ese modo, la nueva ciencia se convertiría en un modelo de interpretación histórica que promueve la modernización industrial en el orbe. 


\section{La crítica a los romanticismos}

El pensamiento filosófico de Abbagnano no puede entenderse sin considerar su constante crítica a los sistemas filosóficos considerados románticos. Sus reflexiones sobre las condiciones epistémicas del conocimiento histórico y sociológico desarrollaron ampliamente dicha oposición. El filósofo italiano consideró al positivismo y al idealismo como sistemas románticos caracterizados por cuatro rasgos principales: (i) Optimismo: la convicción de que la realidad es todo lo que debe ser; (ii) Providencialismo histórico: la idea de la historia como un proceso necesario en el que la razón infinita se manifiesta o se realiza a sí misma; (iii) Tradicionalismo: la exaltación de la tradición y de las instituciones que encarna; y (iv) Titanismo: el culto y la exaltación del infinito (2001b, p. 946). En el presente apartado se propone una interpretación sobre las implicaciones epistémicas de dicha crítica.

Contra la filosofía positivista de Comte, Abbagnano desarrolló en diferentes ensayos una crítica orientada a desmitificar la concepción de razón necesaria en los procesos históricos y sociales. El carácter de esta crítica presenta al menos dos matices. En primer lugar, consideraba que la estructura de la existencia humana, así como las características metodológicas de las ciencias sociales, no permite el descubrimiento de leyes inmutables y necesarias. Como consecuencia de este hecho, pensaba que la sociología y la historia eran incapaces de realizar predicciones infalibles por más rigurosos que fueran sus métodos. En el fondo, ambas caras de la moneda coinciden en un punto crucial: la crítica contra los romanticismos del s. XVIII consistía en evidenciar su carácter determinista e historicista que prescinde del factor de la libertad. La estructura de la existencia, caracterizada por la indigencia y la problematicidad, contemplaría también la libertad individual como la variable que impide que los fenómenos sociales puedan ser descritos mediante leyes inmutables o predicciones infalibles.

La crítica de Abbagnano contra el positivismo parte de una noción de vital importancia para las ciencias sociales. Consideraba que dicho modelo pretendía la erección de una ciencia absoluta de la sociedad en la que se postulaba que los fenómenos sociales podían ser predecibles, como en algunos campos de la física, mediante procesos mecánicos. La razón por la cual considerará esta propuesta como romántica es que el positivismo sostuvo originalmente un modelo determinista que no considera los factores de la libertad, la creatividad y la espontaneidad humanas, así como la problemática interacción ambiente-individuo-sociedad. En el fondo, su crítica se centró en: (1) la interpretación del cambio histórico como proceso necesariamente progresivo y (2) la idea de que el individuo concreto estuviera determinado fatalmente por leyes sociales o históricas infalibles. En este sentido, el determinismo positivista apuntaba a la consolidación de un esquema mecanicista en el seno de las ciencias sociales que Abbagnano no admitía.

La crítica contra el romanticismo positivista, en la filosofía neoilluministica, no se redujo a una mera oposición al providencialismo histórico o al optimismo, sino que también se extendió al campo de las ciencias básicas y la epistemología. Considerando a Laplace como el científico que mejor representaba el espíritu de este movimiento intelectual, Abbagnano rechazó su teoría sobre la absoluta predictibilidad de los fenómenos naturales, apoyándose en las tesis de la física cuántica.

El determinismo causal de Laplace, fuertemente influido por los postulados de Newton, puede considerarse como la extensión ontológica y epistemológica natural de las tesis de la física clásica. Por un lado, dicha física concebía al universo como una máquina regulada por leyes inmutables. Por otro, la naturaleza matemática de tales leyes haría posible la predictibilidad absoluta de los fenómenos. El determinismo del científico francés puede concebirse como un mecanicismo determinista que considera que en la base de todos los fenómenos naturales (orgánicos o inorgánicos) operan las leyes de Newton. En palabras de Laplace:

Una inteligencia que en un momento determinado conociera todas las fuerzas que animan a la Naturaleza, así como la situación respectiva de los seres que la componen, si además fuera lo suficientemente amplia como para someter a análisis tales datos, podría abarcar en una sola fórmula los movimientos de los cuerpos más grandes del universo y los del átomo más ligero; nada le resultaría incierto y tanto el futuro como el pasado estarían presentes ante sus ojos. (1814, p. 243).

Contra la tesis de Laplace, Abbagnano elaboró una estrategia dúplice compuesta de: (i) un argumento físico y (ii) 
un argumento epistémico. En el caso (i), señaló que la física cuántica no concibe a las partículas como cosas en el mismo sentido que se conciben en el mundo macroscópico. Las partículas indicarían un complejo concepto subatómico descrito mediante una determinación matemática (una función), lo que permite calcular la probabilidad de encontrar un determinado resultado en futuras observaciones. El filósofo italiano estaba convencido que las partículas, al constituir la base microscópica de la realidad, eran una mera función matemática y jamás indicó con exactitud sus verdaderas limitaciones en el mundo macroscópico que describe la física clásica. En el caso (ii), indicó que la previsión infalible es imposible, no por la imperfección de los instrumentos de observación o cálculo disponibles, sino porque los sistemas de observación influyen imprevisiblemente sobre los hechos observados (2001, p. 170). Por tanto, su refutación del determinismo positivista estuvo condicionada por la idea de que tanto a nivel físico como a nivel epistémico las únicas categorías explicativas son la probabilidad y la posibilidad.

Los primeros rasgos de la crítica de Abbagnano a los romanticismos pueden rastrearse desde sus primeros trabajos de juventud, centrados en la búsqueda de las fuentes irracionales del pensamiento, así como en el análisis de las implicaciones filosóficas de los nuevos paradigmas de las matemáticas (formalismo y geometrías no euclidianas) y la física cuántica. Como la tesitura de su crítica fue madurando con el tiempo, se pueden rastrear al menos cuatro momentos de vital importancia: (a) el período de su tesis universitaria en el que combatió el logicismo idealista mediante el vitalismo bergsoniano; (b) el período de la física nueva con su análisis sobre las implicaciones epistémicas del principio de indeterminación y de la teoría de la relatividad; (c) el período del existencialismo positivo, con la elaboración de una filosofía enfocada en la estructura de la existencia y en la categoría de la posibilidad de lo posible; y (d) el período neoilluministico, con el empirismo metodológico o condicionalismo epistémico como estrategias racionales de elección que evitan extrapolaciones románticas y que permiten nuevas elecciones en el proceso de investigación.

La constante en la filosofía de Abbagnano fue su inclinación a defender un modelo condicionalista (ni determinista ni indeterminista, ni mecanicista ni relativista) de la estructura de la existencia, así como del conocimiento científico. Su crítica juvenil al logicismo idealista reflejaba la necesidad de reivindicar la legitimidad de las fuerzas vitales como elementos fundamentales de la condición humana. Durante este proceso tuvo una primera aproximación a conceptos esenciales en su filosofía como slancio vitale, impegno, rischio, proggetto, fallibilità, considerados como condiciones o posibilidades vitales que niegan el determinismo. Frente al mecanicismo logicista de los idealistas, el joven Abbagnano recurrió al vitalismo para introducir la cuestión de la problematicidad existencial como uno de los problemas centrales de la existencia humana y, por ende, del pensamiento filosófico.

En el período (b) efectuó una profunda revaloración acerca de las posibles implicaciones epistémicas de los postulados de la física cuántica y la teoría de la relatividad. En su particular interpretación del principio de indeterminación de Heisenberg, recuperó la idea de que en el mundo cuántico operan leyes distintas a las descritas por la física clásica, puesto que existen límites para la experimentación empírica, siendo imposible predecir contemporáneamente la posición y la velocidad de una partícula determinada. De la teoría de Einstein, recuperó la superación de las nociones de referencialidad y contemporaneidad absolutas que están en la base de la física newtoniana. En este período, su crítica al positivismo determinista de Laplace consistió en negar la previsión mecánica e infalible de los fenómenos, para abrazar la previsión probabilística que ofrece la física cuántica.

En el período (c) Abbagnano desarrolló la idea de que la estructura de la existencia podía ser descrita con la categoría posibilidad, mientras que la estructura del conocimiento científico podía serlo con la categoría probabilidad. De ese modo, la dupla posibilidad-probabilidad definiría tanto un esquema ontológico de existencia como un esquema epistemológico de conocimiento. Mientras la estructura de la existencia humana estaría caracterizada por la problematicidad y la coexistencia, en la estructura de la ciencia predominaría la superación de esas condicionantes mediante instrumentos liberatorios siempre perfectibles.

En el período (d), elaboró una concepción de la ciencia entendida como libertad en la que el empirismo 
metodológico o condicionalismo epistémico serían fundamentales. Su crítica al positivismo puso en evidencia el carácter mecanicista-determinista de dicho modelo, negando la presencia del factor libertad en los fenómenos socio-históricos. De tal modo, el positivismo podía considerarse romántico porque reduce la realidad a la manifestación de un principio infinito. En sus palabras: "La característica del positivismo es la romantización de la ciencia: su exaltación como la única guía de la vida individual y social del ser humano, esto es, único conocimiento, única moral y única religión posible” (2001b, p. 836). El empirismo metodológico tendría la misión de establecer una serie de condiciones epistémicas que impidan la exaltación romántica de la ciencia.

En su ensayo L'ultimo Croce e il soggetto della storia, Abbagnano criticó también el idealismo de Hegel y Croce, exponiendo que el fundamento del romanticismo idealista se resuelve en la concepción de la realidad histórica como la infinidad de potencia y de valor de la razón absoluta. La historiografía de Croce es vista como un modelo que rechaza la filosofía del espíritu, al reconocer la independencia del ser y del deber ser como fundamento de la conciencia moral. De este modo, la concepción romántica del idealismo expresaría la necesidad de escapar a un fatalismo radical, en el que la libertad individual no existe ni siquiera como problema filosófico. El romanticismo idealista habría fracasado identificando la libertad con la necesidad histórica.

La crítica de Abbagnano contra los romanticismos del s. XVIII se fundaba en una clara intuición: tales modelos evocan un principio infinito (el espíritu absoluto, el mecanicismo o el determinismo) como fundamento de los procesos históricos y sociales, negando espacio a la posibilidad, a la probabilidad y a la libertad humana. En sus palabras: "El culto y la exaltación del infinito, el contentarse exclusivamente con la infinidad, constituyen los aspectos más sobresalientes del espíritu romántico” (2001b, p. 947). Dentro de este cuadro podrían incluirse los sistemas metodológicos que cumplan con una, o más, de las siguientes condiciones:

i. Que consideren posible formular verdades universales y necesarias

ii. Que crean posible realizar una descripción exhaustiva y total del mundo, mediante la concatenación causalnecesaria de los eventos, los cuales se consideran expresables mediante leyes inmutables de la naturaleza

iii. Que consideren posible describir el cuadro completo, inmutable y necesario de las funciones intelectuales que posibilitan la ciencia como producción intelectual

iv. Que consideren posible que la filosofía sea una disciplina universal y necesaria, estando inspirada en el modelo de la ciencia

v. Que consideren posible que el ser humano pueda alcanzar un grado de seguridad y garantía infalibles respecto a su propio destino en el mundo (1950, pp. 4-5).

Para Abbagnano la principal condición epistémica de las ciencias sociales consistía en incluir el factor de la libertad humana (el riesgo, la problematicidad, la elección, etc.), renunciando a la pretensión de que los fenómenos sociales son procesos gobernados por leyes universales, necesarias y progresivas. La ciencia histórica tendría que recurrir a las fuentes primarias del conocimiento, a datos empíricos como objetos arqueológicos o inscripciones, sin especular sobre nociones románticas como el sentido de la historia. Lo mismo tendría que hacer la sociología, construyendo sus propias categorías de análisis y divorciándose definitivamente de la filosofía y el derecho. Las condiciones epistémicas para la historia y la sociología constituirían una especie de manual propedéutico para la elaboración de sus particulares campos de saber, posibilitando la creación de lenguajes especializados para analizar fenómenos empíricos complejos. La renuncia al romanticismo se podría interpretar, en este sentido, como un primer paso hacia la rigurosidad epistémica tanto para la sociología como para la historia.

\section{Condiciones epistémicas para la investigación histórica}

En la filosofía de Abbagnano la elaboración de las condiciones epistémicas para la investigación histórica estuvo profundamente influida por su crítica a los romanticismos. Rechazando la tesis de que los fenómenos naturales o 
los fenómenos socio-históricos son gobernados por leyes infalibles, se inclinó a construir un modelo de interpretación histórica basado en las nociones de condición, posibilidad y probabilidad. En el presente apartado mostraré las principales características del condicionalismo epistémico, entendido como un marco metodológico que posibilita el diálogo entre distintas disciplinas cuyo objetivo fundamental es el reconocimiento histórico del pasado del hombre y de sus productos más excelentes (1953, p. 220).

En primer lugar, es importante resaltar que la concepción historiográfica de Abbagnano incluye una profunda crítica a las tesis del determinismo social y del determinismo biológico. Considerando al materialismo histórico como el modelo que representa de mejor manera el primer tipo de determinismo, escribió: "El concepto materialista de la historia es una derivación inmediata de la antropología marxista", en la que "el hombre está constituido esencialmente por relaciones sociales objetivas, condicionadas por la forma histórica de la producción y del trabajo" (1967, p. 116). Los argumentos que opuso al determinismo materialista fueron los siguientes:

1. Las personas no están determinadas necesariamente por las relaciones sociales, solamente están condicionadas por aquellas.

2. El campo de las elecciones posibles, entre dos extremos radicales de aceptación pura y simple o de su refutación, constituyen la libertad de la persona.

3. El esquema de las previsiones infalibles y el mito del curso inevitable de la historia deben ser abandonados (1967, p. 119).

En el caso del determinismo biológico, Abbagnano identificó al genetismo como su modelo más representativo y al racismo como su rigurosa extensión ideológica. El romanticismo genetista conduce a sostener que: (a) la superioridad de un individuo es producida necesariamente por su estructura física, y (b) la estructura física del individuo es producida necesariamente por su herencia genética. Este tipo de concepción puede considerarse estrictamente determinista al no considerar la interacción entre herencia genética y el ambiente, como lo sugiere hoy la epigenética. Considerando al gen como la posibilidad de transmisión de características físicas determinadas, y no como una fuerza productiva, el filósofo italiano interpretó la genética en un esquema probabilista mucho más acorde a nuestros tiempos. Las condiciones de manifestación de factores genéticos dependería, en su visión, de: (i) la interacción de los genes entre sí y (ii) la interacción genes-ambiente.

En la historiografía adoptada por Abbagnano, el determinismo (idealista, positivista, materialista o genetista) debía ser abandonado a condición de no promover un paradigma indeterminista. Su modelo de interpretación histórica se funda en la idea de que las condiciones, las posibilidades, la problematicidad y el ejercicio de la libertad son elementos esenciales para un análisis sólidamente fundado del pasado. Cuando se habla de condiciones epistémicas se presume la existencia de un canon de criterios metodológicos o técnicas de atestación que sean preferibles a otras porque posibilitan el análisis de datos empíricamente falsificables o verificables. La evolución de los instrumentos para la medición de magnitudes físicas ofrece un claro ejemplo de cómo el desarrollo de mejores tecnologías permite ofrecer información mucho más detallada y mucho más exacta que la obtenida en generaciones anteriores. El desarrollo de estas condiciones epistémicas incluyen, claramente, la creación de un lenguaje científico propio a cada ciencia, permitiendo que las teorías y las observaciones se retroalimenten constantemente.

En su trabajo sobre el Lavoro storiografico in filosofia, Abbagnano confrontó su modelo de interpretación con el modelo idealista, resaltando sus diferencias. En primer lugar, contrapuso la orientación arqueológica de su propuesta de interpretación con la ofrecida por la orientación filológica del idealismo. Mientras su historiografía obliga al respeto de un canon y al uso de todo el material arqueológico disponible, el idealismo se contentaría con la interpretación de textos. En segundo lugar, resaltó que su historiografía requiere la técnica de la prospectiva histórica, como una condición epistémica de necesario distanciamiento de los objetos a estudiar. De tal modo, las condiciones epistémicas mínimas para la ciencia histórica serían las siguientes: 
1. Disponibilidad del material documentario: textos, biografías, noticias, etc. Prioridad de la historia arqueológica sobre la historia filológica.

\section{Actitud crítica o regla del distanciamiento del objeto de estudio. Se compone de:}

a) La posibilidad de la individuación histórica: Determinar el objeto de estudio de modo inconfundible, como algo que no se repite siguiendo las mismas formas.

b) Relevamiento de la cronología: La colocación de un evento inconfundible en el tiempo.

3. Elaboración de un canon de elecciones historiográficas: La intervención de los intereses en cuestión es legítimo, siempre y cuando no se falsee la historia. El deber ético del historiador consiste en formular, con la mayor claridad posible, las reglas de la investigación mediante:

i. Respeto del material arqueológico

ii. Respeto de la sintaxis lingüística

iii. La limitación del interés a la propuesta de problemas, no de soluciones.

4. Elaboración de canon de las relaciones históricas: Clarificar las relaciones funcionales y cualquier posible confrontación polémica, de reciproco aclaramiento, mediante el material arqueológico disponible. Debe limitarse a asumir la posibilidad de las relaciones, negándose a prescribir anticipadamente las formas y los modos que puede asumir (2001, pp. 180-188).

Las condiciones epistémicas que refiere Abbagnano pueden ser consideradas también como un breve reglamento epistémico y ético para la labor historiográfica, en tanto que establece los comportamientos que posibilitan una interpretación rigurosa u objetiva del pasado. En este caso, la rigurosidad estará íntimamente asociada con la refutación del romanticismo. El cumplimiento de las condiciones epistémicas impedirían cualquier extrapolación injustificada de corte ideológico.

En su trabajo Dewey: esperienza e possibilità, Abbagnano delineó con mayor amplitud algunos de los rasgos de su propuesta metodológica para la historia. Desde su punto de vista "la historia incluye tanto condiciones objetivas como condiciones subjetivas: por un lado, las objetivas se manifiestan mediante las fuerzas y los eventos; por otro, se compone de registros y valoraciones de estos eventos realizadas por algunos hombres” (2001, p. 401). La historiografía para ser rigurosa debía proponerse no solamente describir y registrar la experiencia pasada, sino fundamentalmente sentar las bases para organizar observaciones y experiencias futuras.

Como hemos podido ver hasta aquí, las condiciones epistémicas de la investigación histórica tienen una indudable deuda con la crítica que Abbagnano formuló a los sistemas románticos, considerados normalmente como la expresión de un plan providencial cuyo orden se realiza infalible y progresivamente mediante el acontecer histórico. La razón por la que modelos de esa naturaleza no pueden considerarse razonables es que ningún dato empírico podría permitir una generalización válida sobre el sentido de la historia. Por lo mismo, el abandono de la explicación teleológica, finalista o providencial es una condición necesaria, aunque no suficiente, para analizar a la historia como un proceso en el que confluyen condiciones y libertad. En ese sentido, su historiografía considera que "la historia del hombre sobre la tierra no puede ser entendida como un progreso unilineal y necesario".

Parece bastante probable que la oposición de Abbagnano al determinismo estuviera condicionada por su convicción de que la libertad humana debía asumirse como un factor decisivo en los fenómenos sociales. En este contexto, si la historia aspiraba a convertirse en una ciencia capaz de desarrollar metodologías propias e imparciales, así como técnicas racionales autónomamente rectificables, debía ser capaz de liberarse de los presupuestos y los prejuicios promovidos por los distintos tipos de romanticismo. La ciencia histórica debía renunciar a explicaciones metafísicas como el sentido de la historia. Su labor consistiría en interpretar correctamente los objetos arqueológicos concretos, para ser capaz de individuar espacial y cronológicamente los acontecimientos, así como para ofrecer explicaciones rigurosamente justificadas de los acontecimientos del 
pasado.

\section{Condiciones epistémicas para la investigación sociológica}

Igual que con la historia, Abbagnano consideraba que la sociología debía purificarse de los elementos románticos heredados del positivismo. Este filtro de renuncia al optimismo, al providencialismo y al titanismo fue considerado fundamental. La sociología debería acudir a objetos como documentos, testimonios, datos estadísticos y sociométricos, así como a la observación directa para constituirse como verdadera ciencia. Su modelo es interesante al estar compuesto de modo heterogéneo, reuniendo nociones del existencialismo, el probabilismo, el conductualismo, el institucionalismo y el funcionalismo.

En el período neoilluministico, Abbagnano sostuvo la concepción de que el conocimiento científico se organiza mediante distintos campos, cada uno con su propio lenguaje y con su propia sintaxis, sus propios métodos y sus propias técnicas. Pensó que una de las condiciones epistémicas fundamentales para el desarrollo de la sociología consistía en limitar su propio campo, distinguiéndolo de la ciencia histórica. La forma en la que realizó esta separación fue considerando la perspectiva metodológica. En este sentido, la ciencia histórica fue concebida como un modo de conocimiento enfocado en la individuación de eventos singulares que se reconstruyen en su específica particularidad; mientras que la sociología se enfoca en estudiar las instituciones y las actitudes por su recurrencia (1951, pp. 26-27). A esto habría que agregar también que la historia se enfoca en estudiar hechos del pasado, mientras que la sociología se concentra en interpretar el presente buscando establecer las condiciones de posibles predicciones sobre el futuro.

Mientras la historia analiza fenómenos singulares del pasado que no son generalizables mediante leyes históricas, la sociología analiza la recurrencia de actitudes y relaciones en determinados grupos, por lo que tendría al menos la capacidad de indicar las condiciones que posibilitan un fenómeno social específico. En otras palabras, si la historia no podía hacer uso de la generalización inductiva, la sociología podía aspirar a generalizaciones o predicciones estadísticas.

En su trabajo Per una teoria sociologica generale, Abbagnano planteó algunas categorías fundamentales y algunas condiciones epistémicas para el saber sociológico. La teoría sociológica es concebida como "un grupo organizado de significados lingüísticos, capaz de guiar la investigación experimental y de describir sus resultados". A su vez, los significados lingüísticos son vistos como "todos los términos a los que se les puede encontrar un uso oportuno en función de la investigación experimental o que dependa de ella” (1967, p. 31). Una condición epistémica fundamental consistía en conseguir que la observación empírica y la teoría estuvieran estrechamente vinculadas. En este esquema, una teoría que no hace referencia a la investigación de campo no tiene cabida, puesto que las especulaciones metafísicas no pueden considerarse ciencia. Como lo dice el propio filósofo, el éxito de erigir una sociología científica depende en gran medida de no construir una "metafísica de la sociedad".

La categoría fundamental en la sociología de Abbagnano es la de hechos sociales. Considerados el campo de estudio por excelencia, son definidos como relaciones comunicativas en las que no existe una completa garantía de éxito o seguridad. La sociología tendría la misión de estudiar el carácter uniforme, común y repetible de los fenómenos sociales (las instituciones). Para cumplir su misión debería considerar el factor actitudinal o de atteggiamento, que condiciona el desenlace de las relaciones comunicativas y de la recurrencia de los comportamientos.

Además de la importancia de la creación de un lenguaje propio para el conocimiento sociológico, Abbagnano puso énfasis en su aspecto metodológico. En este sentido, indicó que algunas condiciones epistémicas para el método sociológico son: (a) la observación actitudinal y conductual, (b) la elaboración de un cuadro de soluciones posibles respecto a grupos determinados de actitudes recurrentes, y asignar a cada solución un índice de probabilidad, (c) el descubrimiento de relaciones funcionales en el análisis. 
En el caso de la observación actitudinal y conductual, priorizó el análisis de datos obtenidos empíricamente, rechazando el análisis teórico o especulativo que prescinde de la observación. La sociología, para convertirse en verdadera ciencia, debía cumplir con la condición de que sus datos fueran rigurosamente elaborados mediante metodologías y técnicas de observación controlada. Se trataba, pues, de elaborar un método empírico y crítico que permitiera la explicación de las condiciones que dan vida a los hechos sociales, pero que fuera capaz de autoperfeccionarse.

En segundo lugar, estaba convencido que la filosofía era capaz de ayudar a la sociología a clarificar su propio aparato conceptual con el objetivo de que la sociología pudiera desarrollar su propio empirismo metodológico. En otras palabras, la sociología debía ser capaz de llegar a un punto en el que pudiera perfeccionar autónomamente sus métodos, sus conceptos y sus técnicas. Estos esfuerzos debían cristalizar en estudios que indicarán la probabilidad de comportamientos condicionados, no necesarios.

Finalmente, la identificación de las relaciones funcionales de análisis serían la base para construir un discurso epistémicamente riguroso en el que están presentes: (i) el factor comunicativo (hecho social), (ii) el factor conductual (actitudes e instituciones), (iii) el factor funcional (los grupos sociales) y (iv) el factor de la libertad (la posibilidad de una elección o acción).

La sociología de Abbagnano tiene un fuerte componente probabilístico al considerar que los hechos sociales están condicionados por actitudes e instituciones, pero no determinados fatalmente por leyes infalibles. En su concepción, la sociología debía ser capaz de incluir el factor de la libertad individual, puesto que en todas las actitudes y comportamientos siempre está presente el riesgo. A esto se puede sumar el hecho de que el factor probabilístico es determinante para la fiabilidad de los resultados obtenidos en la investigación sociológica. En otras palabras: "solamente a través de un número suficientemente grande de observaciones particulares, pueden ser puestas a la luz, constatadas y controladas, las uniformidades recurrentes que forman el objeto de la descripción” (1967, p. 42).

Su modelo sociológico incluyó el factor de la libertad humana como un elemento presente en las conductas sociales. Uno de los mejores instrumentos para poder conocer la frecuencia, la recurrencia y la institucionalización de conductas y actitudes sería la observación directa y el uso de instrumentos estadísticos-probabilísticos. Así como en su existencialismo la categoría de la posibilidad de lo posible define la estructura de la existencia, en su sociología la categoría de la probabilidad define el lenguaje en el que pueden describirse los fenómenos sociales.

La sociología de Abbagnano recogió algunos elementos de su existencialismo positivo. La categoría de actitud o atteggiamento puede considerarse el puente que usó para introducir el factor de la libertad individual como un elemento clave en la investigación sociológica. Cuando el filósofo italiano precisaba el significado del término atteggiamento (actitud) recurre a ideas como "forma de ser", "apertura social”, "posibilidad de comunicación y participación”. Parece claro, en este sentido, que consideraba una pretensión legítima apelar a una especie de condicionalismo actitudinal, para sustituir el modelo de causa necesaria del determinismo. De hecho, en su proyecto es evidente que una condición epistémica consistía en abandonar la explicación causal, sustituyéndola por el concepto de condición, puesto que el condicionalismo expone posibilidades delimitadas por un campo de elección medianamente restringido. Las relaciones humanas, vistas de esta manera, debían ser comprendidas como relaciones libres que implican decisiones y elecciones de los miembros que componen los grupos sociales. Por la misma razón, el objeto de la sociología sería describir funcionalmente y probabilísticamente la recurrencia de actitudes y acciones.

En la sociología de Abbagnano el factor actitudinal es vital como condición para la explicación objetiva de las relaciones sociales, así como para entender la institucionalización de los comportamientos y para explicar sociológicamente la creación de valores morales como fenómenos generados por la recurrencia de prácticas sociales específicas. Una teoría sociológica que no incluyera el factor actitudinal estaría condenada a esbozar un determinismo causal incapaz de comprender cómo las elecciones individuales y las condiciones sociales se relacionan complejamente para dar vida a los fenómenos sociales. En otras palabras, la ciencia sociológica tendría 
que renunciar al romanticismo determinista de elaborar previsiones infalibles, creando un modelo condicionalista en el que el concepto de causa: (1) no se asume como fuerza productiva y (2) no implica la univocidad e inevitabilidad del efecto (167, p. 109).

Abbagnano consideraba además que la ciencia sociológica podía ser la llave para comprender fenómenos como la discrepancia de las valoraciones morales en los grupos sociales, así como la disparidad en el uso de las proposiciones morales. Al igual que la psicología y la economía, que lograron constituirse en disciplinas autónomas rechazando a la metafísica, la sociología debería organizarse sobre bases empíricas y conceptuales capaces de autoperfeccionarse. Las condiciones epistémicas de la investigación sociológica debían apuntar a una alianza entre existencialismo, funcionalismo, conductualismo, institucionalismo y técnicas de probabilidad. Solamente así se podría conseguir explicar los fenómenos sociales desterrando la elaboración de una ciencia romántica que elabore leyes deterministas o infalibles de la sociedad.

\section{Conclusiones}

La crítica de Abbagnano a los romanticismos fue vital en su concepción de las condiciones epistémicas para la sociología y la historia. Dicha crítica implicaba fundamentalmente la refutación del determinismo, pero es importante distinguirla de cualquier tipo de indeterminismo. Desde su punto de vista, el giro paradigmático a asumir era de carácter condicionalista-probabilista. Su propuesta incluía, por tanto, el uso de técnicas de observación, datos empíricos y herramientas matemáticas para describir la frecuencia de los fenómenos sociales, sin descartar el factor de la libertad humana. En este sentido, su crítica es parcialmente satisfactoria, al reivindicar un modelo multicondicional que es óptimo para el análisis de fenómenos complejos.

Las condiciones epistémicas para la historia pueden enumerarse de la siguiente manera:

1. El abandono del romanticismo

2. Abandono del determinismo porque:

a) Las personas no están determinadas, sino condicionadas

b) Existen las elecciones posibles, dentro de un horizonte medianamente predecible

c) No tiene sentido hablar de previsiones infalibles o de un sentido intrínseco en la historia.

3. Uso del material arqueológico como fuente primaria.

4. La adopción de la técnica de la perspectiva histórica.

5. El método histórico podría ser esquematizado del modo siguiente:

O Observación (material arqueológico)

D Determinación objeto de estudio (tiempo y espacio)

$\mathrm{H}$ Elaboración canon de elecciones historiográficas en función de O

E Explicación o canon de relaciones históricas.

$\mathrm{O} \rightarrow \mathrm{D} \rightarrow \mathrm{H} \rightarrow \mathrm{O} \rightarrow \mathrm{E}$

Por su parte, las condiciones epistémicas para la sociología contemplarían:

1. El abandono del romanticismo determinista. La explicación mecánica es sustituida por la tríada condiciónposibilidad-probabilidad.

2. Distinción del objeto de estudio. 
3. Evitar la creación de una metafísica de la sociedad, mediante una forma de vinculación de la teoría con la práctica en la que la segunda prevalece sobre la primera.

4. Es posible construir un empirismo metodológico que puede ser esquematizado de la forma siguiente:

O Observación: i. Comunicativa ii. Conductual iii. Funcional iv. Posibilidad de elección

S Elaboración de soluciones posibles respecto a acciones recurrentes en grupos determinados

P Asignación de índice de posibilidad a cada solución

F Descubrimiento de relaciones funcionales

$\mathrm{O} \rightarrow \mathrm{S} \rightarrow \mathrm{O} \rightarrow \mathrm{P} \rightarrow \mathrm{F}$

El esfuerzo sincrético de Abbagnano, que consistió en unir en una sola teoría sociológica los factores que componen a los fenómenos sociales, parece bastante apropiado para el análisis de fenómenos complejos. En su favor, se puede decir que evita una explicación unilateral de los fenómenos sociales, eligiendo la descripción de probabilidades estadísticas, comportamientos y funciones recurrentes, pero considerando los factores del riesgo y la problematicidad de los propios fenómenos como factores condicionantes.

El empirismo metodológico de Abbagnano sugería que la filosofía podía auxiliar a las ciencias sociales a encontrar su objeto de estudio, su lenguaje y sus métodos. Su propuesta consideraba imprescindible que ese empirismo condujera a la independencia de la sociología y de la historia. En ese sentido, su crítica al idealismo y al positivismo estaba enfocada en evitar la erección de una romántica metafísica del infinito, en la que la libertad, la posibilidad, el riesgo y la probabilidad no tuvieran cabida.

Los estudios más actuales en las ciencias sociales parecen haber dado la razón, al menos en parte, a Abbagnano. Primeramente, porque la creación de metodologías empíricas requiere la renuncia a motivaciones románticometafísicas. En segundo lugar, porque las metodologías más fiables recurren actualmente a modelos de carácter condicional-probabilístico para la interpretación de los fenómenos. Finalmente, porque las metodologías empíricas capaces de autocorregirse dan clara evidencia de la perfectibilidad de los instrumentos de verificación y falsificación. Algunos de los casos en los que se cumplen esas condiciones son: (a) el cálculo matemático del riesgo; (b) los estudios de elección racional (c) la sociología y la política comparada; (d) las ciencias cognitivas y (e) la estadística.

La lista de condiciones epistémicas de Abbagnano podría, sin embargo, ser enriquecida si se agregarán las siguientes premisas para el análisis de fenómenos complejos:

1. No es posible realizar la previsión exacta del estado futuro de un fenómeno socio-histórico, considerando única y exclusivamente sus estados precedentes.

2. La magnitud de los fenómenos socio-históricos no se corresponde necesariamente con la magnitud de las causas verificables.

3. Los fenómenos socio-históricos son muy sensibles a las acciones de ciertos actores y grupos, así como a las condiciones iniciales.

4. Los fenómenos socio-históricos implican relaciones causales bidireccionales o multidireccionales.

5. La incidencia exacta de los factores que componen un fenómeno socio-histórico no se puede identificar en todos los casos.

6. Una teoría para los fenómenos socio-históricos es más completa cuantos más elementos considere en el entrecruzamiento de la información, y cuanta mayor capacidad posea para prever los factores que intervienen en un fenómeno concreto. 
7. Una teoría para los fenómenos socio-históricos requiere: a) el uso de instrumentos empíricos fiables para la obtención de datos; b) el uso de criterios epistémicos para evitar derivar información no consistente con los datos obtenidos; c) el uso de un lenguaje cuantitativo claro y preciso (o en su defecto, un lenguaje cualitativo que permita clasificar y comparar); y, d) el uso de potentes herramientas de análisis que fomenten la racionalidad crítica y la colectivización del conocimiento.

\section{Bibliografia}

Abbagnano, N. (1923). Le sorgenti irrazionali del pensiero. Genova: Perella.

Abbagnano, N. (1934). La fisica nuova: fondamenti di una teoria della scienza. Napoli: Guida.

Abbagnano, N. (1939). La struttura dell'esistenza. Napoli: Paravia.

Abbagnano, N. (1947). Filosofia, religione, scienza. Torino: Taylor.

Abbagnano, N. (1947). Fondamenti logici della scienza. Torino: Francesco de Silva.

Abbagnano, N. (1948). Esistenzialismo positivo: due saggi. Torino: Taylor.

Abbagnano, N. (1950). Saggi di critica delle scienze. Torino: Franceso de Silva.

Abbagnano, N. (1956). Possibilità e libertà. Torino: Taylor.

Abbagnano, N. (1967). Problemi di sociologia. Torino: Taylor.

Abbagnano, N. (2001). Scritti neoilluministici. Torino: Unione tipografico-editrice torinese.

Abbagnano, N. (2001 b). Dizionario filosofico. Torino: UTET.

Bobbio, N. et al. (1982). La cultura filosofica italiana dal 1945 al 1980. Napoli: Guida editori.

Comte, A. (2004). Curso de filosofía positiva. Buenos Aires: Libertador.

Croce, B. (1902). Estetica come scienza dell' espressione e linguistica generale. Milano-Palermo-Napoli: Sandron.

Croce, B. (1909). Logica come scienza del concetto puro. Bari: Laterza.

Cuozzo e Riconda et al. (2008). Le due Torino. Primato della religione o primato della politica?. Torino: Trauben.

Durst, M. (1998). Gentile e la filosofia nell’Enciclopedia Italiana. Roma: Pellicani.

Fornero, G. (1980). Sull'esistenzialismo positivo: Abbagnano e Gentile. Rivista critica di storia della Filosofia, 35(4), 416-437. 
Fornero, G. (1984). Concetto e critica del romanticismo ottocentesco nel pensiero di Nicola Abbagnano. Rivista di storia della filosofia, 39(3), 551-570.

Fornero, G. (1995). Il concetto di storia della filosofia nella riflessione teorica e metodologica di Nicola Abbagnano. In Una filosofia dell'uomo. Atti del Convegno in memoria di Nicola Abbagnano (a cura di Cacciatore e Cantillo) (pp. 153-162). Comune di Salerno.

Gentile, G. (1920). Teoria generale dello spiritu come atto puro. Laterza: Edizione Trabant.

Giannini, G. (1956). L'esistenzialismo positivo di Nicola Abbagnano. Brescia: Morcelliana.

Hegel, F. (1974). Enciclopedia de las ciencias filosóficas. México: Juan Pablos editor.

Hegel, F. (2003). Fenomenología del espíritu. México: FCE.

Langiulli, N. (1992). Possibility, necessity, and existence: Abbagnano and his predecessors. Philadelphia: Temple University Press.

Laplace (1814). Essai philosophique sur les probabilités. Paris: Courcier.

Miglio, B. et al. (2002). Nicola Abbagnano. Un itinerario filosofico. Bologna: Il Mulino.

Mori, M. (2010). Libertà, necessità, determinismo. Bologna: Il Mulino.

Paolini, M. (1998). Consuntivo storico e filosofico sul “Centro di studi metodologici” di Torino (1940-1979). Genova: Pantograf.

Pasini e Rolando (1991). Il neoilluminismo italiano. Cronache di filosofia (1953-1962). Milano: Il Saggiatore.

Primerano, G. (2009). La prospettiva pedagogica di Nicola Abbagnano. Roma: Aracne.

Remo, C. (1947). La dittadura del idealismo. Il Politecnico, 37, 3-6.

Rossi e Viano et al. (2004). Le città filosofiche. Per una geografia della cultura filosofica italiana del Novecento. Bologna: Il Mulino.

Semerari (1969). Il neoilluminismo filosofico italiano. En Esperienze del pensiero moderno (pp. 273-93). Urbino.

Simona, M. (1962). La notion de liberté dans l'existentialisme positif de Nicola Abbagnano. Fribourg: Editions universitaires.

Tega, W. (2010). Impegno per la ragione. Il caso del neoilluminismo. Bologna: Il Mulino.

Viano, C. (1996). La filosofia italiana del Novecento. Bologna: Il Mulino.

Viano, C. (2007). Stagione filosofiche. La filosofia del novecento fra Torino e l'Italia. Bologna: Il Mulino. 